\title{
Numerical simulation of Rapid Additive Forging (RAF) process
}

\author{
Lionel Depradeux ${ }^{1 *}$, Corentin Robitaille*, Gilles Duval ${ }^{1 * *}$, Luc Eckenfelder**, Camille Locatelli** \\ * EC2-Modélisation, Villeurbanne, France \\ ** PRODWAYS Group \\ 1 : Corresponding authors : lionel.depradeux@ec2-modelisation.fr, g.duval@prodways.com
}

\section{Summary:}

The Rapid Additive Forging (RAF) process is a Direct Energy Deposition (DED) Additive Manufacturing (AM) process, based on the deposition of a Titanium alloy on a substrate plate. This process has been developed for the production of Titanium parts of aeronautic components. In this study, a Finite Element (FE) numerical simulation methodology has been established to perform a fast analysis of the RAF process, including full 3D-transient thermal-metallurgical and mechanical numerical simulations. Thus, residual stresses and distortions caused by the process can be estimated. Different modelling strategies have been compared in order to find a balance between computation time and accuracy. Analyses include the effects of phase transformations in the Titanium alloy. First analyses have been performed on a simple geometry of welding wall. The influences of the material activation modelling strategy on the thermal and mechanical results have been investigated. The effects of phase transformations on residual stresses and distortions are also discussed.

Then a specimen with a more complex geometry has been considered in the analysis, including the effect of different deposition paths. A full 3D simulation of the whole deposition process has been compared with several simplified computation procedures, including a reduction of the number of layers considered in the simulation.

Keywords: Additive manufacturing, numerical simulation, welding wall, Titanium alloy, phase transformations, residual stresses, distortions

\section{Introduction}

Additive Manufacturing (AM) by welding-like metal deposition process appears very attractive to manufacture pieces of large scale, especially for aircraft components. In order to produce Titanium parts for aeronautic components, a specific process, the Rapid Additive Forging (RAF) process has been developed by Prodways. This is a Direct Energy Deposition (DED) AM process, based on the deposition of molten titanium wire on a substrate plate. However, many aspects of this process, that involves complex thermal, metallurgical and mechanical phenomena are not scientifically well established and require a deeper understanding. Moreover, some aspects of the process need to be optimized. For that purpose, numerical simulation is an interesting alternative to experimental design campaigns, so as to understand the effects of weld parameters, clamping conditions, preheating, etc..., especially on residual stresses and distortions. Recent advances in the field of numerical weld simulation allow using this kind of analysis in an industrial context [1]. Numerical simulations remain nevertheless difficult, due to the 
large number of passes involved. In addition, the mechanical constitutive equations of the material are generally difficult to calibrate over a large temperature range. Additionally, for materials that exhibit solid-state transformations (such as ferritic steels or titanium alloys), these effects must also be taken into account [1-3]. In this study, a modelling of the RAF process has been developed, in order to better understand the involved phenomena, and to investigate the effects of various parameters.

With this model, various numerical simulations of metallic deposition have been performed, on different test-cases, in order to calibrate the simulation methodology. The first test case considers a wall made of Titanium alloy, with a unidirectional deposition for each weld pass. The model includes thermal, metallurgical, and mechanical effects that take place during the process. Then a more complex case has been considered (piece with $\mathrm{H}$ like geometry) for the simulation, considering different kinds of material (Nickel-based alloys or austenitic steels), so as to investigate the effects of path deposition and simplified simulation procedures. All the simulations are performed with the FE code Abaqus (Simulia).

\section{Titanium alloy welding wall: experiment and simulation}

\section{- Test case characteristics}

The first test case analyzed in this study is the deposition of a 25 layers high $(55 \mathrm{~mm})$, two pass wide, $200 \mathrm{~mm}$ long TA6V alloy wall, considering the RAF deposition procedure. The substrate plate dimensions are $\left(300^{\prime} 60^{\prime} 15\right) \mathrm{mm}^{3}$. During the weld, the substrate is clamped on a platform on the rear and the front sides of the substrate (figure 1). Each layer is composed of 2 passes. All the weld passes are deposited along the same direction. At the end of the welding (i.e. end of final cooling), the clamping conditions are released, and the final distortion (deflection of the substrate) is measured.

In order to analyze the weld residual stresses and distortions, a non-linear transient thermal simulation is first performed so as to compute the temperature field. Then a subsequent metallurgical and mechanical computation is carried out, taking into account the temperaturedependent mechanical material properties, as well as the effects of metallurgical evolution.

- Non-linear thermal transient simulation

For the thermal analysis, a simple volumetric power heat source density, moving along the weld, was considered in the bead. The total net power was directly estimated from the weld parameters. Although more sophisticated double ellipsoidal models [5] were also initially considered, it has been proven that the heat source shape had little effect on the weld residual stresses and distortions, when considering a similar net heat power. Temperature dependent thermo-physical properties, taken from literature [2-3], have been considered. Convective and radiative thermal boundary conditions have also been considered on the surfaces of the workpiece. After each weld deposit, the piece is allowed to cool down to a temperature lower than $70^{\circ} \mathrm{C}$. 


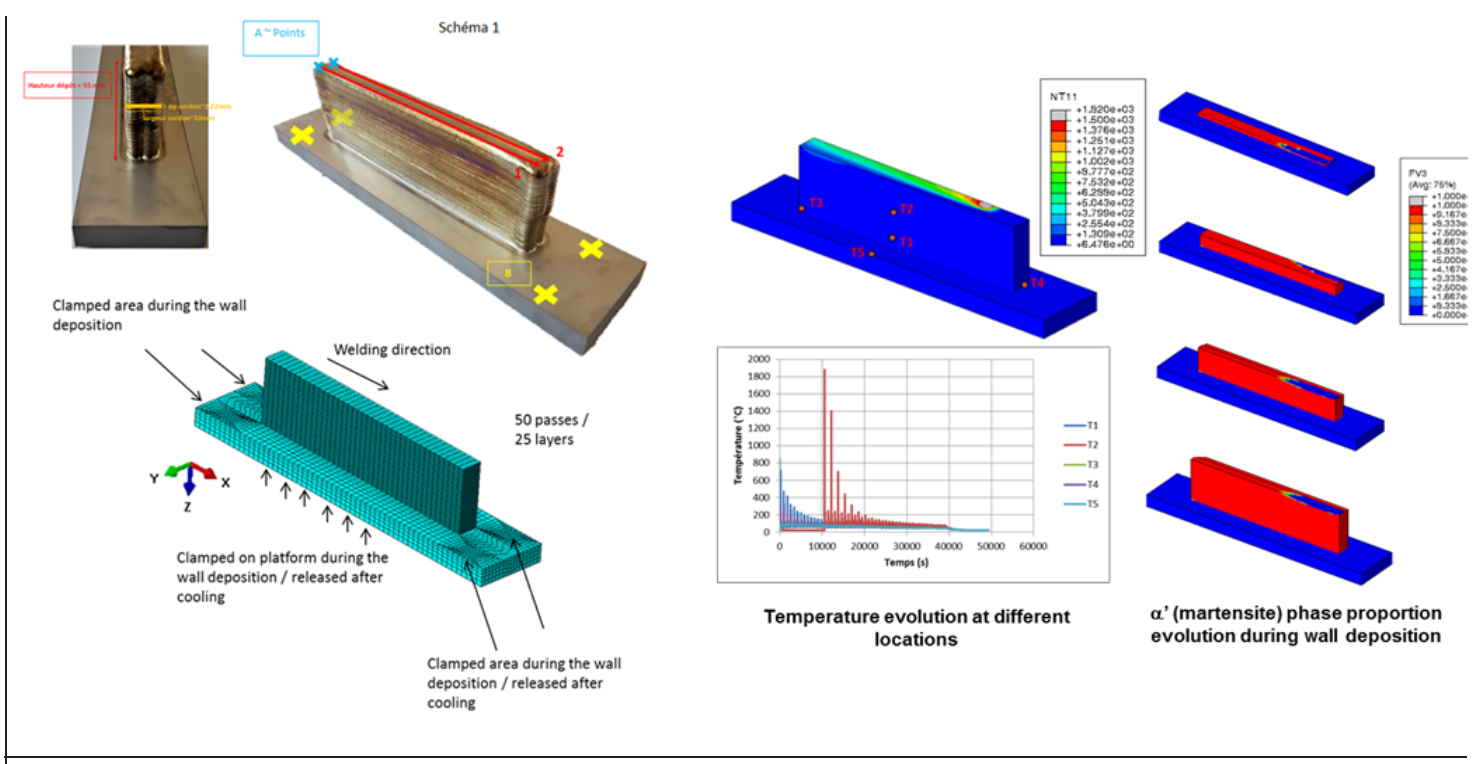

Figure 1 : Clamping conditions of the welding Wall mesh, and computed thermal and metallurgical evolutions during the wall deposition

Different strategies of metal activation during the deposit were considered, using a "birth element" technique: First, a bead by bead activation (as frequently considered in multi-passes weld simulation), and then a more realistic progressive activation along the weld line for each pass. This second method is more realistic, as element ahead of the heat source are not present in the model. However, the activation method has been found to have very little effect on the results in this case, although the first method leads to simulation approximatively 2 times faster than the second one.

\section{- Metallurgical model}

The solid-phase state transformations that take place in the Titanium alloy during welding were taken into account in the analysis, with a simplified procedure. Three metallurgical phases have been included in the analysis: a, b (at high temperature) and a' (martensitic phase, during cooling). The proportions (\%) of phases were considered, in order to include, in the mechanical analysis, the effects of volumetric changes during transformations. These effects can either superimpose or counterbalance the thermal dilatation, and then affect the weld residual stresses and distortions. In this case, given the very high cooling rate $\left(>100^{\circ} \mathrm{C} / \mathrm{s}\right)$, only martensitic phase is expected in the welding wall. For the martensitic transformation, a Koistinen-Marburger kinetic was considered between the Ms/Mf temperatures.

\section{- Mechanical analysis}

For the mechanical analysis, an elasto-plastic Von Mises constitutive equation was considered for the different phases. Temperaturedependent material properties were taken from [3]. At this stage of the study, visco-plasticity was not taken into account, and elasto-plastic properties were extrapolated at high temperature considering the strain rate. The final effects of phase transformations on stresses and distortions were investigated, by comparing simulations, with or without including these solid state transformations. The mechanical computation also includes two stages: first, the computation of the weld deposit of the 50 passes ; then, the boundary conditions are released so as to study the stress redistribution, as the workpiece re-equilibrates, and the deflection starts to develop. Figure 2 shows the residual 
stresses obtained at two instants: at the end of the wall construction (after cooling) when the clamping conditions are still considered, and after the release of these boundary conditions, leading to maximum plate deflection. Figure 2 compares the computed and measured deflection of the plate at the end of the computation, showing a very good agreement.

The simulation ignoring the phase transformations leads to a similar final deflection, but with significantly higher residual stresses in the wall (figure 2). Though the stresses distribution is qualitatively globally similar for both computations, the stress intensity is notably affected by the solid state transformations. Residual stress measurements are currently ongoing in order to qualify the simulation assumptions. However, first comparisons with residual stress measurement from the literature show a very promising agreement.

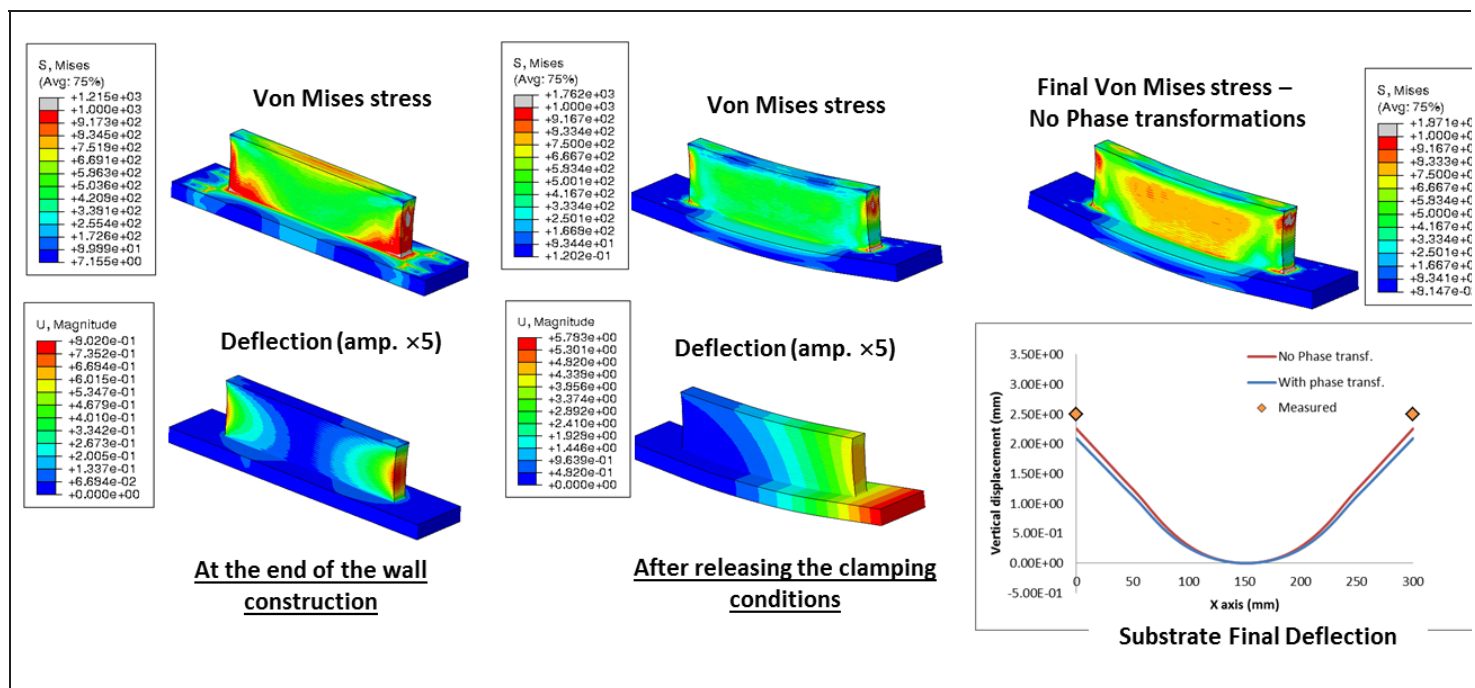

Figure 2 : Computed weld residual stresses and distortions, at the end of the cooling, before and after the release of the clamping conditions, an comparison with experimental deflections

\section{More complex case: $\mathrm{H}$ workpiece}

After the methodology calibration on the welding wall, a more complex test-case was considered, with a complex deposition path. The dimensions of the substrate are $\left(300^{\prime} 200^{\prime} 50\right) \mathrm{mm}^{3}$. The materials considered are either nickel based alloy or austenitic stainless steel (yield stress $\sim 300 \mathrm{MPa}$ at room temperature). Numerical simulations have been performed using the same methodology as the one described in the previous section. No solid phase state transformation occurs in such materials, so the analysis is somehow simpler. The thermal computation is performed with a similar triangular heat source, with a progressive activation of the weld metal along the weld line. The initial CAD of the model is subdivided into several weld "spots", and their successive activations during the computation are automatically taken into account, according to a list of points that describes the deposition path. Then the mechanical simulation is performed, considering temperature-dependent mechanical properties, with an elasto-plastic Von Mises constitutive equation and linear kinematic hardening. The numerical simulation is then used to investigate the effects of path deposition on residual stresses. It was found that, for this special test case, the path deposition procedure has little effects on the residual stresses, but more significant effects on distortions (figure 3 ). 


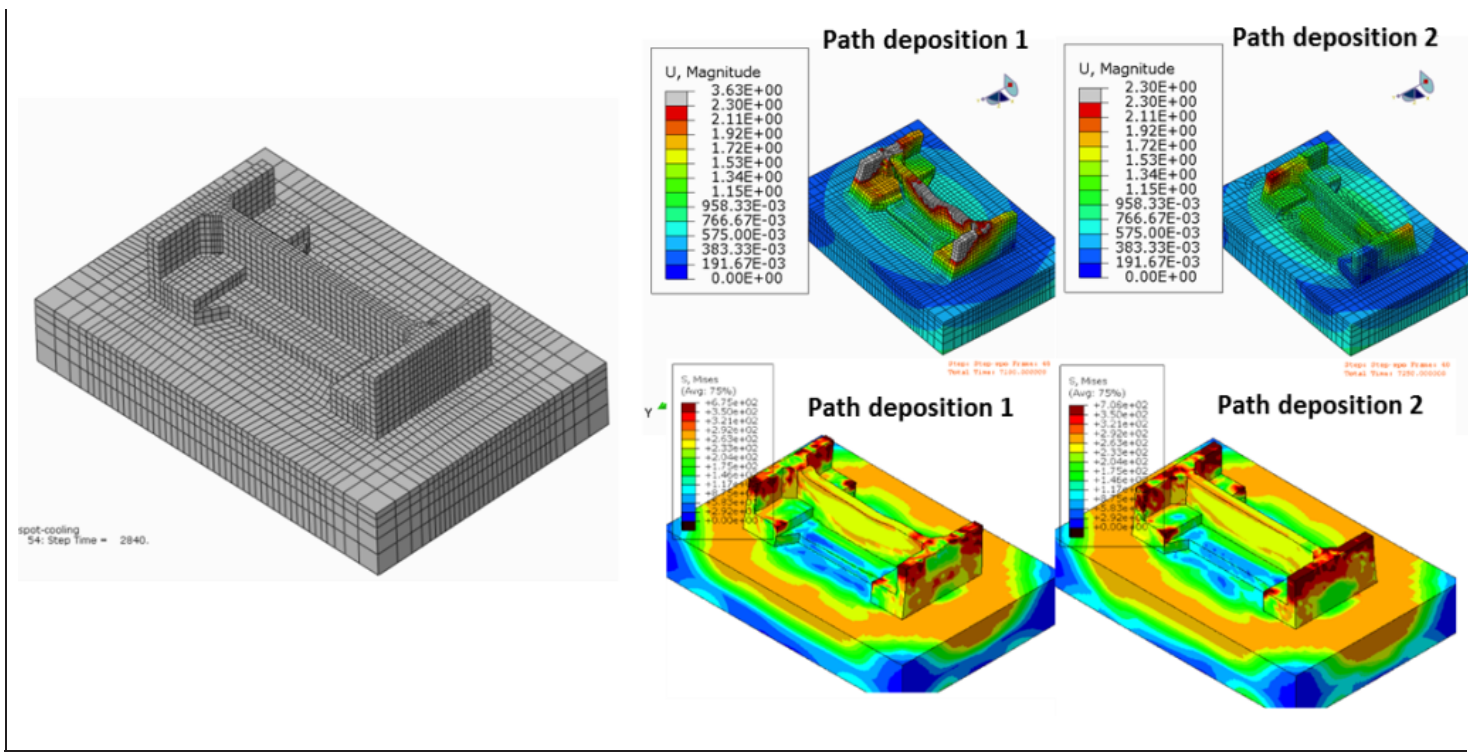

Figure $3: \mathrm{H}$ workpiece mesh and residual stresses and distortion computed for different welding paths

\section{Simplified methodology investigation}

Though acceptable from the industrial point of view, full 3D simulation of the complete deposit procedure remains rather time-consuming. Therefore, it seems particularly interesting to investigate simplified simulation techniques. In additive manufacturing of welding wall, it is observed that the first beads generate high stresses and distortions, whereas the effects of subsequent passes are less pronounced. Moreover, it has also been noticed that the last weld passes also have a significant impact on the final residual stress fields. This kind of considerations has led to modelling strategies that do not include all the passes in the numerical analysis, but only a reduced number of them [4]. This approach looks promising for additive manufacturing, for which a very high number of passes is to be considered. Figure 4 shows a comparison between the weld residual stresses obtained by the computation of the complete deposit of the weld beads, and a simulation that considers only the first and the last layers. Despite some discrepancies in the intermediary layers, the results are rather consistent in a large part of the workpiece. Though this kind of simplified methodology needs obviously to be improved (by including some selected intermediary layers), it appears very attractive, as the second simulation is approximatively 10 times faster than the first one. Further analyses are currently ongoing so as to optimize the procedure. 


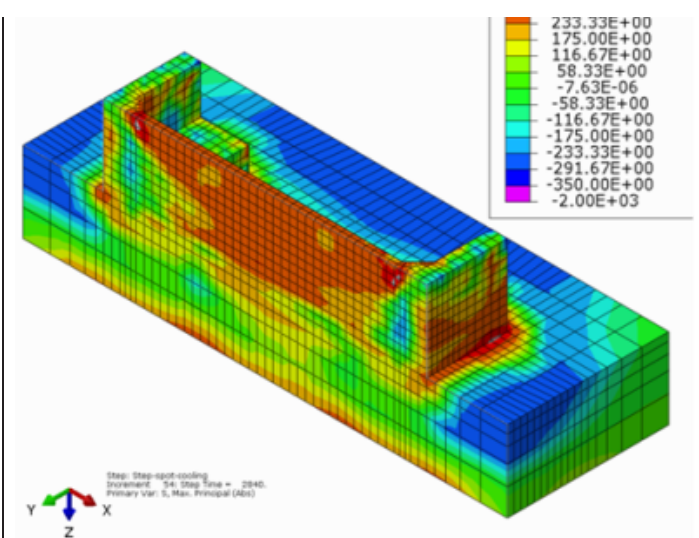

Max. principal stress in the middle plane - full computation of all layers

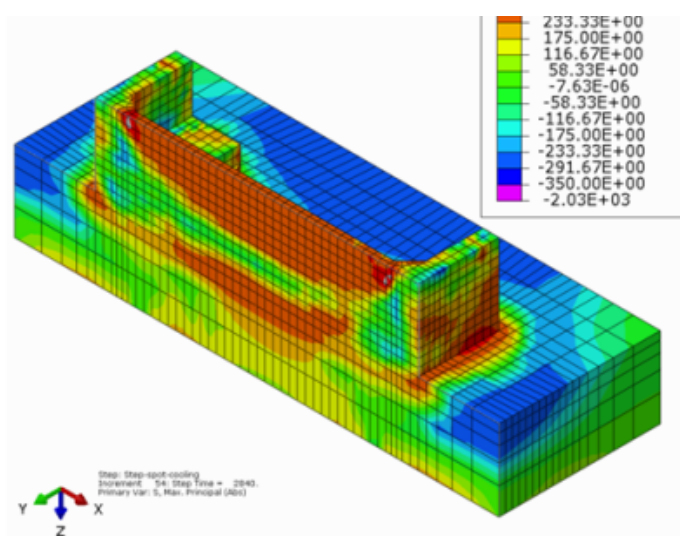

Max. principal stress in the middle plane computation of only the 1st and last layer

Figure 4 : Comparison of weld residual stresses in the central plane of the piece between a full computation of all the layers, and a simplified computation strategy including only the $1^{\text {st }}$ and last layers

\section{References}

[1] L.E Lindgren , Finite element modelling and simulation of welding, Part 1 to 3, Journal of thermal stresses, 2001, 24, pp.141-192, 195$131,305-334$

[2] Y. Robert, Simulation numérique du soudage du TA6V par laser YAG impulsionnel : caractérisation expérimentale et modélisation des aspects thermomécaniques associés à ce procédé, Mines Paris Tech PhD thesis, 2007

[3] A. Longuet, Modélisation du procédé de projection laser - Application au Ti-6Al-4V, Mines Paris Tech PhD thesis, 2010

[4] L. Depradeux, F. Rossillon, A time saving method to compute multi-pass weld residual stresses, paper $n^{\circ}$ PVP2013-97239, Proceedings of the ASME 2013 Pressure Vessels \& Piping Conference - PVP 2013, July 14-18, Paris 2013

[5] J. Goldak, A. Chakravati, M. Bibby,, A new finite element model for welding heat sources, Metallurgical Transactions, 1984, 15B, pp. 299305

[6] J Ahn, E. He, L. Chen, R.C Wimpory, J-P. Dear, C.M. Davies, Prediction and measurment of residual stresses and distorsion in fibre laser welded Ti-6Al-4V considering phase transformation, Material and Design 115 (2017) 441-457 